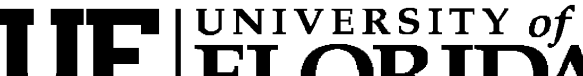 FLORIDA \\ IFAS Extension
}

\section{Management of Insect and Mite Resistance in Ornamental Crops ${ }^{1}$}

James F. Price, Elzie McCord, Jr., and Curtis Nagle ${ }^{2}$

Resistance of arthropods to crop management chemicals has been problematic since the early era of synthetic organic pesticides. During the 1970s and early 1980s leafminer (Liriomyza trifolii) outbreaks heavily damaged herbaceous ornamental crops such as chrysanthemum, gypsophila, aster, and marigold in fields, shade houses and greenhouses. Several effective insecticides including organophosphates, carbamates, pyrethroids, and a triazine were identified for leafminer control during the outbreak; however, control was short-lived as the leafminer developed resistance to each insecticide.

Poor performance of pesticides does not always indicate pest resistance. Such factors as pesticide degradation in storage, hydrolysis in acid or alkaline preparations, applications to an incorrect life stage, or other inadequate application procedures may contribute to poor control.

\section{Definition of Resistance}

Pest populations can be susceptible or resistant to a pesticide. Resistance occurs when a formerly susceptible pest population becomes significantly less susceptible to a pesticide and degradation of the pesticide or improper application is not a factor. Pesticide resistance is a population-based phenomenon in which the genetic composition shifts and the population becomes dominated by individuals possessing genes that confer resistance.

\section{Establishment of Resistance}

Resistant populations are protected from formerly effective pesticides through one or more means. For example, resistant pests may: (1) deactivate (break down), (2) sequester (safely store within their bodies), (3) avoid, or (4) excrete the toxin from their bodies more effectively, (5) have an altered target site that will not accumulate the toxin, or (6) reduce the permeability by the toxin through their exoskeletons ("shells").

Individuals within a susceptible pest population often vary in their level of susceptibility; however, the non-susceptible type occurs only very rarely. When a pesticide is applied repeatedly, the susceptible pests die and the resistant ones survive, mate with other survivors and reproduce. Some of their offspring inherit the parents' characteristic for survival. Upon additional applications, the more

1. This is document ENY-843 (IN715), a publication of the Department of Entomology and Nematology, Florida Cooperative Extension Service, IFAS, University of Florida. Publication date: November 2007. Please visit the EDIS Website at http://edis.ifas.ufl.edu.

2. James F. Price, associate professor, Gulf Coast Research and Education Center; Elzie McCord, Jr., associate professor, Dept. of Biological Sciences, New College of Florida; Curtis Nagle, biological scientist, Gulf Coast Research and Education Center, Cooperative Extension Service, IFAS, University of Florida, Gainesville, FL 32611.

The Institute of Food and Agricultural Sciences (IFAS) is an Equal Opportunity Institution authorized to provide research, educational information and other services only to individuals and institutions that function with non-discrimination with respect to race, creed, color, religion, age, disability, sex, sexual orientation, marital status, national origin, political opinions or affiliations. U.S. Department of Agriculture, Cooperative Extension Service, University of Florida, IFAS, Florida A. \& M. University Cooperative Extension Program, and Boards of County Commissioners Cooperating. Larry Arrington, Dean 
susceptible of the offspring within the remaining population die and the less susceptible ones survive, mate with other survivors and produce more similar offspring. Further applications additionally select for the resistant individuals until that form (genotype) is common. The population then is regarded as resistant and the effectiveness of the pesticide is lost.

\section{Resistance Management}

Resistance can develop rapidly with pests that have many generations per year, when multiple generations are exposed to a pesticide, and when new individuals do not move into a treated area to dilute the frequency of the resistant genes. Ornamentals production, especially in greenhouses, often incorporates a combination of these factors that contribute to resistance and account for some of the leafminer problems experienced decades ago.

The main objectives of resistance management programs in ornamentals production should be to minimize the number of exposures of pests to pesticides with a similar mode of action and to use non-chemical approaches to arthropod management. (Mode of action is the specific activity of the toxin that results in the death of the pest. For instance, one mode of action is to inhibit mitochondrial complex I electron transport. This causes a failure of the pest to produce energy in affected cells and to die.)

Repeated exposures to a pesticide are the primary drivers of resistance but much can be done to manage pests by means other than chemicals. Care can be taken to rotate crops, establish new ones only after the older crops have been removed, use pest resistant species and varieties, set pest-free transplants, conserve and release natural enemies, etc. Pest-specific tactics are available for particular situations such as elimination of excessive moisture in order to kill fungus gnats in greenhouses.

Crops should be scouted on a regular, frequent schedule and pesticide applications should be made only when pest densities approach economic injury levels. When pesticide use is required, products should be rotated among the different modes of action indicated on many modern product labels. A list of modes of action can be found by selecting "MoA Classification Scheme" at the Insecticide
Resistance Action Committee Website: http://www. irac-online.org/Crop_Protection/MoA.asp.

Tables 1-3 present a mode of action summary for insecticides and miticides intended for ornamentals production in Florida. Sound rotation plans often recommend pesticides of one mode of action for one pest generation and a pesticide of a different mode of action for another generation. If multiple pesticide applications are required, rotations should continue through all practical modes of action before returning to a previously used one. The use of certain unique products with known general modes of action (such as soaps and oils) is unlikely to result in pest resistance and no codes are assigned. These can be used without regard to a rotation plan for resistance management.

When pesticides are used, it is important to assure that fresh, fully potent pesticides are prepared and applied in accordance with label directions. Aqueous pesticidal preparations should be adjusted to near neutral $\mathrm{pH}(\mathrm{pH}$ 7.0) or as specified by the label. Sprayer calibration, nozzle condition and pressure, and spray placement must be correct. Applications also should be timed and directed to contact the most susceptible life stage of the pest.

\section{Conclusion}

Episodes of pest resistance to popular pesticides can cause crop yield losses, crop quality reduction, added control costs, increased human exposure to toxins, and degradation of the environment. These consequences can be alleviated if resistance management is practiced throughout Florida's ornamentals industry. If growers minimize pesticide application by depending more on biological and cultural pest control measures, and reduce pest exposures to pesticides with identical modes of action, then resistance can become a rare phenomenon. 
Table 1. Mode of action of insecticides and miticides registered for use in Florida's ornamental production (presented by active ingredient). (Insecticide Resistance Action Committee mode of action classification codes version 5.3).

\begin{tabular}{|c|c|c|}
\hline Active Ingredient (Common Name) & Trade Name Examples & Mode of Action Code \\
\hline 1,3-dichloropropene & Telone & \\
\hline abamectin & $\begin{array}{l}\text { Avid } \\
\text { Lucid }\end{array}$ & 6 \\
\hline acephate & $\begin{array}{l}\text { Orthene } \\
\text { Tame/Orthene }^{a}\end{array}$ & 1B \\
\hline acequinocyl & Shuttle & $20 \mathrm{~B}$ \\
\hline acetamiprid & TriStar & $4 \mathrm{~A}$ \\
\hline azadirachtin & $\begin{array}{l}\text { Aza-Direct } \\
\text { Azatin }\end{array}$ & $18 B$ \\
\hline azinphos-methyl & Guthion & 1B \\
\hline Bacillus thuringiensis aizawai & Xentari & 11B1 \\
\hline Bacillus thuringiensis israelensis & Gnatrol & $11 \mathrm{~A} 1$ \\
\hline Bacillus thuringiensis kurstaki & $\begin{array}{l}\text { Dipel } \\
\text { Javelin }\end{array}$ & 11B2 \\
\hline Beauveria bassiana & $\begin{array}{l}\text { Botanigard } \\
\text { Mycotrol } \\
\text { Naturalis }\end{array}$ & \\
\hline bifenazate & Floramite & 25 \\
\hline bifenthrin & $\begin{array}{l}\text { Allectus }^{\mathrm{b}} \\
\text { Attain } \\
\text { Talstar }\end{array}$ & 3 \\
\hline buprofezin & Talus & 16 \\
\hline carbaryl & Sevin & $1 \mathrm{~A}$ \\
\hline carbofuran & Furadan & $1 \mathrm{~A}$ \\
\hline chlorfenapyr & Pylon & 13 \\
\hline chlorpyrifos & $\begin{array}{l}\text { Duraguard } \\
\text { Duraplex } \\
\text { Dursban }\end{array}$ & $1 \mathrm{~B}$ \\
\hline clarified hydrophobic extract of neem oil & Triact & \\
\hline clofentezine & Ovation & $10 \mathrm{~A}$ \\
\hline clothianidin & Celero & $4 \mathrm{~A}$ \\
\hline cryolite & $\begin{array}{l}\text { Kryocide } \\
\text { Prokil Cryolite }\end{array}$ & $9 \mathrm{~A}$ \\
\hline cyfluthrin & $\begin{array}{l}\text { Decathlon } \\
\text { Discus }^{b} \\
\text { Duraplex }^{d}\end{array}$ & 3 \\
\hline cyromazine & Citation & 17 \\
\hline deltamethrin & Deltagard & 3 \\
\hline diazinon & Diazinon & $1 \mathrm{~B}$ \\
\hline
\end{tabular}


Table 1. Mode of action of insecticides and miticides registered for use in Florida's ornamental production (presented by active ingredient). (Insecticide Resistance Action Committee mode of action classification codes version 5.3).

\begin{tabular}{|c|c|c|}
\hline Active Ingredient (Common Name) & Trade Name Examples & Mode of Action Code \\
\hline diflubenzuron & $\begin{array}{l}\text { Adept } \\
\text { Dimilin }\end{array}$ & 15 \\
\hline dimethoate & Dimethoate & $1 \mathrm{~B}$ \\
\hline dinotefuran & Safari & $4 \mathrm{~A}$ \\
\hline disulfoton & Di-Syston & $1 \mathrm{~B}$ \\
\hline endosulfan & Thionex & $2 A$ \\
\hline esfenvalerate & Asana & 3 \\
\hline ethoprop & Mocap & $1 \mathrm{~B}$ \\
\hline etoxazole & Tetrasan & $10 \mathrm{~B}$ \\
\hline fenbutatin oxide & ProMite & $12 \mathrm{~B}$ \\
\hline fenoxycarb & $\begin{array}{l}\text { Award Fire Ant Bait } \\
\text { Preclude }\end{array}$ & 7B \\
\hline fenpropathrin & $\begin{array}{l}\text { Tame } \\
\text { Tame/Orthene }\end{array}$ & 3 \\
\hline fenpyroximate & Akari & 21 \\
\hline fipronil & Chipco Choice & $2 \mathrm{~B}$ \\
\hline flonicamid & Aria & $9 \mathrm{C}$ \\
\hline halofenozide & Mach 2 & $18 \mathrm{~A}$ \\
\hline hexythiazox & Hexygon & $10 \mathrm{~A}$ \\
\hline hydramethylnon & Amdro & $20 \mathrm{~A}$ \\
\hline imidacloprid & $\begin{array}{l}\text { Allectus }^{\dagger} \\
\text { Discus }^{c} \\
\text { Marathon } \\
\text { Merit }\end{array}$ & $4 \mathrm{~A}$ \\
\hline iron phosphate & Sluggo & \\
\hline kaolin & Surround & \\
\hline lambda-cyhalothrin & Scimitar & 3 \\
\hline malathion & Malathion & 1B \\
\hline metaldehyde & $\begin{array}{l}\text { Deadline } \\
\text { Prozap }\end{array}$ & \\
\hline metam-sodium & Vapam & \\
\hline methidathion & Supracide & 1B \\
\hline methiocarb & Mesurol & $1 \mathrm{~A}$ \\
\hline methyl bromide & 67-33 Preplant Soil Fumigant & $8 \mathrm{~A}$ \\
\hline naled & Dibrom & $1 \mathrm{~B}$ \\
\hline novaluron & Pedestal & 15 \\
\hline oxydemeton-methyl & MSR Spray Concentrate & 1B \\
\hline permethrin & $\begin{array}{l}\text { Ambush } \\
\text { Astro }\end{array}$ & 3 \\
\hline
\end{tabular}


Table 1. Mode of action of insecticides and miticides registered for use in Florida's ornamental production (presented by active ingredient). (Insecticide Resistance Action Committee mode of action classification codes version 5.3).

\begin{tabular}{|c|c|c|}
\hline Active Ingredient (Common Name) & Trade Name Examples & Mode of Action Code \\
\hline phosmet & Imidan & $1 \mathrm{~B}$ \\
\hline piperonyl butoxide & $\begin{array}{l}\text { Diatect }^{\mathrm{g}} \\
\text { Pyrenone }^{\mathrm{g}} \\
\text { Pyreth-It }\end{array}$ & $27 \mathrm{~A}$ \\
\hline $\begin{array}{l}\text { polyhedral occlusion bodies of the nuclear } \\
\text { polyhedrosis virsus of Spodoptera exugia }\end{array}$ & Spod-X & \\
\hline potassium salts of fatty acids & $\begin{array}{l}\text { AllPro Insecticidal Soap } \\
\text { M-Pede }\end{array}$ & \\
\hline propargite & $\begin{array}{l}\text { Omite } \\
\text { Ornamite }\end{array}$ & $12 \mathrm{C}$ \\
\hline pymetrozine & Endeavor & $9 \mathrm{~B}$ \\
\hline pyrethrin & $\begin{array}{l}\text { Diatect }^{\text {h }} \\
\text { PyGanic } \\
\text { Pyrellin } \\
\text { Pyrenone }^{\text {h }} \\
\text { Pyreth-It }^{\text {h }}\end{array}$ & 3 \\
\hline pyridaben & Sanmite & 21 \\
\hline pyriproxyfen & Distance & $7 \mathrm{D}$ \\
\hline refined petroleum distillate & Ultra-Fine Oil & \\
\hline rotenone & Pyrellin $^{g}$ & 21 \\
\hline s-kinoprene & Enstar II & $7 \mathrm{~A}$ \\
\hline s-methoprene & Extinguish & $7 \mathrm{~A}$ \\
\hline spinosad & $\begin{array}{l}\text { Conserve } \\
\text { Entrust } \\
\text { Justice Fire Ant Bait } \\
\text { Spintor }\end{array}$ & 5 \\
\hline spiromesifen & Judo & 23 \\
\hline tau-fluvalinate & Mavrik & 3 \\
\hline tebufenozide & $\begin{array}{l}\text { Confirm } \\
\text { Mimic }\end{array}$ & $18 \mathrm{~A}$ \\
\hline thiamethoxam & Flagship & $4 \mathrm{~A}$ \\
\hline \multicolumn{3}{|c|}{$\begin{array}{l}{ }^{a} \text { This product also contains fenpropathrin, see mode of action code } 3 \text { in table } 2 . \\
{ }^{b} \text { This product also contains imidacloprid, see mode of action code } 4 \mathrm{~A} \text { in table } 2 \text {. } \\
{ }^{c} \text { This product also contains cyfluthrin, see mode of action code } 3 \text { in table } 2 . \\
{ }^{d} \text { This product also contains chlorpyrifos, see mode of action code } 1 \mathrm{~B} \text { in table } 2 \text {. } \\
{ }^{e} \text { This product also contains acephate, see mode of action code } 1 \mathrm{~B} \text { in table } 2 \text {. } \\
{ }^{f} \text { This product also contains bifenthrin, see mode of action code } 3 \text { in table } 2 \text {. } \\
{ }^{g} \text { This product also contain pyrethirn, see mode of action code } 3 \text { in table } 2 \text {. } \\
{ }^{n} \text { This product also contain piperonyl butoxide, see mode of action code } 27 \mathrm{~A} \text { in table } 2 . \\
{ }^{i} \text { This product also contains rotenone, see mode of action code } 21 \text { in table } 2 \text {. }\end{array}$} \\
\hline
\end{tabular}


Table 2. Mode of action of insecticides and miticides registered for use in Florida's ornamental production (presented by mode of action code). (Insecticide Resistance Action Committee mode of action classification codes version 5.3).

\begin{tabular}{|c|c|c|}
\hline Mode of Action Code & Active Ingredient (Common Name) & Trade Name Examples \\
\hline & 1,3-dichloropropene & Telone \\
\hline & Beauveria bassiana & $\begin{array}{l}\text { Botanigard } \\
\text { Mycotrol } \\
\text { Naturalis }\end{array}$ \\
\hline & clarified hydrophobic extract of neem oil & Triact \\
\hline & iron phosphate & Sluggo \\
\hline & kaolin & Surround \\
\hline & metaldehyde & $\begin{array}{l}\text { Deadline } \\
\text { Prozap }\end{array}$ \\
\hline & metam-sodium & Vapam \\
\hline & $\begin{array}{l}\text { polyhedral occlusion bodies of the nuclear } \\
\text { polyhedrosis virus of Spodoptera exugia }\end{array}$ & Spod-X \\
\hline & potassium salts of fatty acids & $\begin{array}{l}\text { AllPro Insecticidal Soap } \\
\text { M-Pede }\end{array}$ \\
\hline & refined petroleum distillate & Ultra-Fine Oil \\
\hline \multirow[t]{3}{*}{$1 \mathrm{~A}$} & carbaryl & Sevin \\
\hline & carbofuran & Furadan \\
\hline & methiocarb & Mesurol \\
\hline \multirow[t]{12}{*}{ 1B } & acephate & $\begin{array}{l}\text { Orthene } \\
\text { Tame/Orthene }^{a}\end{array}$ \\
\hline & azinphos-methyl & Guthion \\
\hline & chlorpyrifos & $\begin{array}{l}\text { Duragard } \\
\text { Duraplex }^{a} \\
\text { Dursban }\end{array}$ \\
\hline & diazinon & Diazinon \\
\hline & dimethoate & Dimethoate \\
\hline & disulfoton & Di-Syston \\
\hline & ethoprop & Mocap \\
\hline & malathion & Malathion \\
\hline & methidathion & Supracide \\
\hline & naled & Dibrom \\
\hline & oxydemeton-methyl & MSR Spray Concentrate \\
\hline & phosmet & Imidan \\
\hline $2 \mathrm{~A}$ & endosulfan & Thionex \\
\hline $2 \mathrm{~B}$ & fipronil & Chipco Choice \\
\hline
\end{tabular}


Table 2. Mode of action of insecticides and miticides registered for use in Florida's ornamental production (presented by mode of action code). (Insecticide Resistance Action Committee mode of action classification codes version 5.3).

\begin{tabular}{|c|c|c|}
\hline Mode of Action Code & Active Ingredient (Common Name) & Trade Name Examples \\
\hline \multirow[t]{9}{*}{3} & bifenthrin & $\begin{array}{l}\text { Allectus }^{\mathrm{b}} \\
\text { Attain } \\
\text { Talstar }\end{array}$ \\
\hline & cyfluthrin & $\begin{array}{l}\text { Decathlon } \\
\text { Discus }^{b} \\
\text { Duraplex }^{c}\end{array}$ \\
\hline & deltamethrin & Deltagard \\
\hline & esfenvalerate & Asana \\
\hline & fenpropathrin & $\begin{array}{l}\text { Tame } \\
\text { Tame/Orthene }^{c}\end{array}$ \\
\hline & lambda-cyhalothrin & Scimitar \\
\hline & permethrin & $\begin{array}{l}\text { Ambush } \\
\text { Astro }\end{array}$ \\
\hline & pyrethrin & $\begin{array}{l}\text { Diatect }^{d} \\
\text { PyGanic } \\
\text { Pyrellin }^{\mathrm{e}} \\
\text { Pyrenone }^{d} \\
\text { Pyreth-It }^{d}\end{array}$ \\
\hline & tau-fluvalinate & Mavrik \\
\hline \multirow[t]{5}{*}{$4 \mathrm{~A}$} & acetamiprid & TriStar \\
\hline & clothianidin & Celero \\
\hline & dinotefuran & Safari \\
\hline & imidacloprid & $\begin{array}{l}\text { Allectus }^{a} \\
\text { Discus }^{a} \\
\text { Marathon } \\
\text { Merit }\end{array}$ \\
\hline & thiamethoxam & Flagship \\
\hline 5 & spinosad & $\begin{array}{l}\text { Conserve } \\
\text { Entrust } \\
\text { Justice Fire Ant Bait } \\
\text { Spintor }\end{array}$ \\
\hline 6 & abamectin & $\begin{array}{l}\text { Avid } \\
\text { Lucid }\end{array}$ \\
\hline \multirow[t]{2}{*}{$7 \mathrm{~A}$} & s-kinoprene & Enstar II \\
\hline & s-methoprene & Extinguish \\
\hline $7 \mathrm{~B}$ & fenoxycarb & $\begin{array}{l}\text { Award Fire Ant Bait } \\
\text { Preclude }\end{array}$ \\
\hline $7 \mathrm{D}$ & pyriproxyfen & Distance \\
\hline $8 \mathrm{~A}$ & methyl bromide & 67-33 Preplant Soil Fumigant \\
\hline $9 \mathrm{~A}$ & cryolite & $\begin{array}{l}\text { Kryocide } \\
\text { Prokil Cryolite }\end{array}$ \\
\hline
\end{tabular}


Table 2. Mode of action of insecticides and miticides registered for use in Florida's ornamental production (presented by mode of action code). (Insecticide Resistance Action Committee mode of action classification codes version 5.3).

\begin{tabular}{|c|c|c|}
\hline Mode of Action Code & Active Ingredient (Common Name) & Trade Name Examples \\
\hline $9 \mathrm{~B}$ & pymetrozine & Endeavor \\
\hline $9 \mathrm{C}$ & flonicamid & Aria \\
\hline \multirow[t]{2}{*}{$10 \mathrm{~A}$} & clofentezine & Ovation \\
\hline & hexythiazox & Hexygon \\
\hline $10 \mathrm{~B}$ & etoxazole & Tetrasan \\
\hline $11 \mathrm{~A} 1$ & Bacillus thuringiensis israelensis & Gnatrol \\
\hline 11B1 & Bacillus thuringiensis aizawai & Xentari \\
\hline 11B2 & Bacillus thuringiensis kurstaki & $\begin{array}{l}\text { Dipel } \\
\text { Javelin }\end{array}$ \\
\hline $12 \mathrm{~B}$ & fenbutatin oxide & ProMite \\
\hline $12 \mathrm{C}$ & propargite & $\begin{array}{l}\text { Omite } \\
\text { Ornamite }\end{array}$ \\
\hline 13 & chlorfenapyr & Pylon \\
\hline \multirow[t]{2}{*}{15} & diflubenzuron & $\begin{array}{l}\text { Adept } \\
\text { Dimilin }\end{array}$ \\
\hline & novaluron & Pedestal \\
\hline 16 & buprofezin & Talus \\
\hline 17 & cyromazine & Citation \\
\hline \multirow[t]{2}{*}{$18 \mathrm{~A}$} & halofenozide & Mach 2 \\
\hline & tebufenozide & $\begin{array}{l}\text { Confirm } \\
\text { Mimic }\end{array}$ \\
\hline $18 \mathrm{~B}$ & azdirachtin & $\begin{array}{l}\text { Aza-Direct } \\
\text { Azatin }\end{array}$ \\
\hline $20 \mathrm{~A}$ & hydramethylnon & Amdro \\
\hline $20 \mathrm{~B}$ & acequinocyl & Shuttle \\
\hline \multirow[t]{3}{*}{21} & fenpyroximate & Akari \\
\hline & pyridaben & Sanmite \\
\hline & rotenone & Pyrellin $^{a}$ \\
\hline 23 & spiromesifen & Judo \\
\hline 25 & bifenazate & Floramite \\
\hline $27 \mathrm{~A}$ & piperonyl butoxide & $\begin{array}{l}\text { Diatect }^{\mathrm{a}} \\
\text { Pyrenone }^{\mathrm{a}} \\
\text { Pyreth-It }^{\mathrm{a}}\end{array}$ \\
\hline \multicolumn{3}{|c|}{$\begin{array}{l}\text { a This product also contains an active ingredient with the mode of action code } 3 . \\
\mathrm{b} \text { This product also contains an active ingredient with the mode of action code } 4 \mathrm{~A} \text {. } \\
{ }^{\mathrm{c}} \text { This product also contains an active ingredient with the mode of action code } 1 \mathrm{~B} \text {. } \\
{ }^{\mathrm{d}} \text { This product also contains an active ingredient with the mode of action code } 27 \mathrm{~A} \text {. } \\
\mathrm{e} \text {. }\end{array}$} \\
\hline
\end{tabular}


Table 3. Mode of action of insecticides and miticides registered for use in Florida's ornamental production (presented by trade name). (Insecticide Resistance Action Committee mode of action classification codes version 5.3).

\begin{tabular}{|c|c|c|}
\hline Trade Name Examples & Active Ingredient (Common Name) & Mode of Action Code \\
\hline 67-33 Preplant Soil Fumigant & methyl bromide & $8 \mathrm{~A}$ \\
\hline Adept & diflubenzuron & 15 \\
\hline Akari & fenpyroximate & 21 \\
\hline Allectus & imidacloprid \& bifenthrin & $4 A \& 3$ \\
\hline AllPro Insecticidal Soap & potassium salts of fatty acids & \\
\hline Ambush & permethrin & 3 \\
\hline Amdro & hydramethylnon & $20 \mathrm{~A}$ \\
\hline Aria & flonicamid & $9 \mathrm{C}$ \\
\hline Asana & esfenvalerate & 3 \\
\hline Astro & permethrin & 3 \\
\hline Attain & bifenthrin & 3 \\
\hline Avid & abamectin & 6 \\
\hline Award Fire Ant Bait & fenoxycarb & 7B \\
\hline Aza-Direct & azadirachtin & $18 \mathrm{~B}$ \\
\hline Azatin & azadirachtin & $18 \mathrm{~B}$ \\
\hline Botanigard & Beauveria bassiana & \\
\hline Celero & clothianidin & $4 \mathrm{~A}$ \\
\hline Chipco Choice & fipronil & 2B \\
\hline Citation & cyromazine & 17 \\
\hline Confirm & tebufenozide & $18 \mathrm{~A}$ \\
\hline Conserve & spinosad & 5 \\
\hline Deadline & metaldehyde & \\
\hline Decathlon & cyfluthrin & 3 \\
\hline Deltagard & deltamethrin & 3 \\
\hline Diatect & pyrethrin \& piperonyl butoxide & $3 \& 27 A$ \\
\hline Diazinon & diazinon & $1 \mathrm{~B}$ \\
\hline Dibrom & naled & $1 \mathrm{~B}$ \\
\hline Dimethoate & dimethoate & $1 \mathrm{~B}$ \\
\hline Dimilin & diflubenzuron & 15 \\
\hline Dipel & Bacillus thuringiensis kurstaki & $11 \mathrm{~B} 2$ \\
\hline Di-Syston & disulfoton & $1 \mathrm{~B}$ \\
\hline Discus & imidacloprid \& cyfluthrin & $4 \mathrm{~A} \& 3$ \\
\hline Distance & pyriproxyfen & $7 \mathrm{D}$ \\
\hline Duragard & chlorpyrifos & $1 \mathrm{~B}$ \\
\hline Duraplex & cyfluthrin \& chlorpyrifos & $3 \& 1 B$ \\
\hline
\end{tabular}


Table 3. Mode of action of insecticides and miticides registered for use in Florida's ornamental production (presented by trade name). (Insecticide Resistance Action Committee mode of action classification codes version 5.3).

\begin{tabular}{|c|c|c|}
\hline Trade Name Examples & Active Ingredient (Common Name) & Mode of Action Code \\
\hline Dursban & chlorpyrifos & $1 \mathrm{~B}$ \\
\hline Endeavor & pymetrozine & $9 \mathrm{~B}$ \\
\hline Enstar II & s-kinoprene & $7 \mathrm{~A}$ \\
\hline Entrust & spinosad & 5 \\
\hline Extinguish & s-methoprene & $7 \mathrm{~A}$ \\
\hline Flagship & thiamethoxam & $4 \mathrm{~A}$ \\
\hline Floramite & bifenazate & 25 \\
\hline Furadan & carbofuran & $1 \mathrm{~A}$ \\
\hline Gnatrol & Bacillus thuringiensis israelensis & $11 \mathrm{~A} 1$ \\
\hline Guthion & azinphos-methyl & 1B \\
\hline Hexygon & hexythiazox & $10 \mathrm{~A}$ \\
\hline Imidan & phosmet & $1 \mathrm{~B}$ \\
\hline Javelin & Bacillus thuringiensis kurstaki & 11B2 \\
\hline Judo & spiromesifen & 23 \\
\hline Justice Fire Ant Bait & spinosad & 5 \\
\hline Kryocide & cryolite & $9 A$ \\
\hline Lucid & abamectin & 6 \\
\hline M-Pede & potassium salts of fatty acids & \\
\hline Mach 2 & halofenozide & $18 \mathrm{~A}$ \\
\hline Malathion & malathion & $1 \mathrm{~B}$ \\
\hline Marathon & imidacloprid & $4 \mathrm{~A}$ \\
\hline Mavrik & tau-fluvalinate & 3 \\
\hline Merit & imidacloprid & $4 \mathrm{~A}$ \\
\hline Mesurol & methiocarb & $1 \mathrm{~A}$ \\
\hline MSR Spray Concentrate & oxydemeton-methyl & 1B \\
\hline Mimic & tebufenozide & $18 \mathrm{~A}$ \\
\hline Mocap & ethoprop & $1 \mathrm{~B}$ \\
\hline Mycotrol & Beauveria bassiana & \\
\hline Naturalis & Beauveria bassiana & \\
\hline Omite & propargite & $12 \mathrm{C}$ \\
\hline Ornamite & propargite & $12 \mathrm{C}$ \\
\hline Orthene & acephate & $1 \mathrm{~B}$ \\
\hline Ovation & clofentezine & $10 \mathrm{~A}$ \\
\hline Pedestal & novaluron & 15 \\
\hline Preclude & fenoxycarb & 7B \\
\hline Prokil Cryolite & cryolite & $9 A$ \\
\hline
\end{tabular}


Table 3. Mode of action of insecticides and miticides registered for use in Florida's ornamental production (presented by trade name). (Insecticide Resistance Action Committee mode of action classification codes version 5.3).

\begin{tabular}{|c|c|c|}
\hline Trade Name Examples & Active Ingredient (Common Name) & Mode of Action Code \\
\hline ProMite & fenbutatin oxide & $12 \mathrm{~B}$ \\
\hline Prozap & metaldehyde & \\
\hline PyGanic & pyrethrin & 3 \\
\hline Pyrellin & pyrethrin \& rotenone & $3 \& 21$ \\
\hline Pyrenone & piperonyl butoxide \& pyrethrin & $27 \mathrm{~A} \& 3$ \\
\hline Pyreth-It & piperonyl butoxide \& pyrethrin & $27 A \& 3$ \\
\hline Pylon & chlorfenapyr & 13 \\
\hline Safari & dinotefuran & $4 \mathrm{~A}$ \\
\hline Sanmite & pyridaben & 21 \\
\hline Scimitar & lambda-cyhalothrin & 3 \\
\hline Sevin & carbaryl & $1 \mathrm{~A}$ \\
\hline Shuttle & acequinocyl & $20 \mathrm{~B}$ \\
\hline Sluggo & iron phosphate & \\
\hline Spintor & spinosad & 5 \\
\hline Spod-X & $\begin{array}{l}\text { polyhedral occlusion bodies of the nuclear } \\
\text { polyhedrosis virus of Spodoptera exugia }\end{array}$ & \\
\hline Supracide & methidathion & $1 \mathrm{~B}$ \\
\hline Surround & kaolin & \\
\hline Talstar & bifenthrin & 3 \\
\hline Talus & buprofezin & 16 \\
\hline Tame & fenpropathrin & 3 \\
\hline Tame/Orthene & fenpropathrin \& acephate & $3 \& 1 \mathrm{~B}$ \\
\hline Telone & 1,3-dichloropropene & \\
\hline Tetrasan & etoxazole & $10 \mathrm{~B}$ \\
\hline Thionex & endosulfan & $2 \mathrm{~A}$ \\
\hline Triact & clarified hydrophobic extract of neem oil & \\
\hline TriStar & acetamiprid & $4 \mathrm{~A}$ \\
\hline Ultra-Fine Oil & refined petroleum distillate & \\
\hline Vapam & metam-sodium & \\
\hline Xentari & Bacillus thuringiensis aizawai & 11B1 \\
\hline
\end{tabular}

